\title{
Very large thermal rectification in ferromagnetic insulator-based superconducting tunnel junctions
}

Cite as: Appl. Phys. Lett. 116, 192601 (2020); https://doi.org/10.1063/5.0010148

Submitted: 07 April 2020 . Accepted: 28 April 2020 . Published Online: 11 May 2020

(iD) F. Giazotto, and (i) F. S. Bergeret

\section{COLLECTIONS}

F This paper was selected as Featured
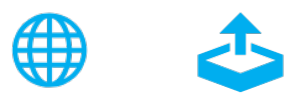

\section{ARTICLES YOU MAY BE INTERESTED IN}

Electron spin resonance with up to 20 spin sensitivity measured using a superconducting flux qubit

Applied Physics Letters 116, 194001 (2020); https://doi.org/10.1063/1.5144722

Developing silicon carbide for quantum spintronics

Applied Physics Letters 116, 190501 (2020); https://doi.org/10.1063/5.0004454

Thermal metamaterials for radiative plus conductive heat flow control

Applied Physics Letters 116, 191902 (2020); https://doi.org/10.1063/5.0007574

\section{Challenge us.}

What are your needs for periodic signal detection?

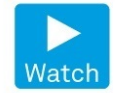

Zurich

- Instruments

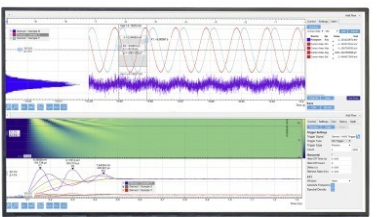

( 


\title{
Very large thermal rectification in ferromagnetic insulator-based superconducting tunnel junctions
}

Cite as: Appl. Phys. Lett. 116, 192601 (2020); doi: 10.1063/5.0010148

Submitted: 7 April 2020 - Accepted: 28 April 2020 .

Published Online: 11 May 2020

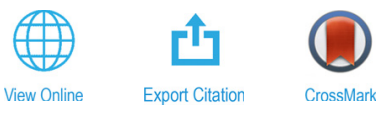

F. Giazotto ${ }^{1, a)}$ (D) and F. S. Bergeret ${ }^{2,3, b)}$ iD

\author{
AFFILIATIONS \\ ${ }^{7}$ NEST Istituto Nanoscienze-CNR and Scuola Normale Superiore, I-56127 Pisa, Italy \\ ${ }^{2}$ Centro de Fisica de Materiales (CFM-MPC), Centro Mixto CSIC-UPV/EHU, Manuel de Lardizabal 5, \\ 20018 Donostia-San Sebastian, Spain \\ ${ }^{3}$ Donostia International Physics Center (DIPC), Manuel de Lardizabal, 4, 20018, Donostia San Sebastian, Spain \\ a) Author to whom correspondence should be addressed: francesco.giazotto@sns.it \\ ${ }^{b)}$ Electronic mail: fs.bergeret@csic.es
}

\begin{abstract}
We investigate electronic thermal rectification in ferromagnetic insulator-based superconducting tunnel junctions. Ferromagnetic insulators coupled to superconductors are known to induce sizable spin splitting in the superconducting density of states and also lead to efficient spin filtering if used as tunnel barriers. The combination of spin splitting and spin filtering is shown to yield a substantial amount of self-amplification of the electronic heat diode effect due to breaking of the electron-hole symmetry in the system, which is added to the thermal asymmetry of the junction. Large spin splitting and large spin polarization ( $\gtrsim 90 \%)$ can potentially lead to thermal rectification efficiencies exceeding $\sim 5 \times 10^{4} \%$ for realistic parameters in a suitable temperature range, thereby outperforming up to a factor of $\sim 250$, the heat diode effect achievable with conventional superconducting tunnel junctions. These results are relevant for improved control of heat currents in innovative phase-coherent caloritronic nanodevices and for enhanced thermal management of quantum circuits at the nanoscale.
\end{abstract}

Published under license by AIP Publishing. https://doi.org/10.1063/5.0010148

A thermal rectifier, or a heat diode, ${ }^{1,2}$ is a device in which the heat current depends on the sign and the amplitude of the temperature gradient imposed across it. The implementation of efficient heat diodes would represent a breakthrough in the realization of improved thermal circuits for cooling, ${ }^{3}$ thermal isolation, energy harvesting, radiation sensing, and several other applications. ${ }^{4}$ Recently, the control of thermal transport at the nanoscale has been attracting great interest. ${ }^{3-6}$ From the theoretical side, strong effort has been put to conceive thermal rectification setups dealing with phonons, ${ }^{7-10}$ electrons, ${ }^{6,11-20}$ and photons. ${ }^{21}$ Experimentally, promising results were obtained so far in the context of electronic ${ }^{22-24}$ and phononic ${ }^{25-27}$ heat transport. Rectification of electronic heat currents has been studied in several types of tunneling junctions between different materials such as, for instance, normal metals, ${ }^{17}$ Josephson junctions, ${ }^{18}$ and superconductor-normal metal structures. ${ }^{6,19}$ In all cases, heat rectification stems from thermal asymmetry of the structure in the direction of the current flow. In conventional superconducting tunnel junctions, this asymmetry together with the highly non- linear temperature dependence of the density of states leads to a heat rectification up to $\sim 800 \%$ in Josephson junctions. ${ }^{18}$

In this Letter, we study thermal rectification effects in systems based on ferromagnetic insulator (FI) superconducting tunnel junctions. The combination of spin splitting and spin polarization induced by FIs breaks the electron-hole symmetry of the electronic transport. This electron-hole symmetry breaking (EHSB) together with the nonlinear temperature dependence of the superconducting spectral properties leads to an unrivaled heat diode effect with a suitable choice of realistic parameters. Specifically, a rectification efficiency exceeding $\sim 5 \times 10^{4} \%$ can be obtained, outperforming up to a factor of $\sim 250$ that is achievable in conventional superconducting tunnel junctions. These results could be relevant for improved thermal isolation of cryogenic quantum circuitry at the nanoscale.

Figure 1(a) schematizes the generic system under investigation, which consists of two electronic reservoirs (either superconducting or normal metallic) residing at temperature $T_{L, R}$. The density of states $N_{L, R}$ depends on the temperature and applied exchange field. The two 

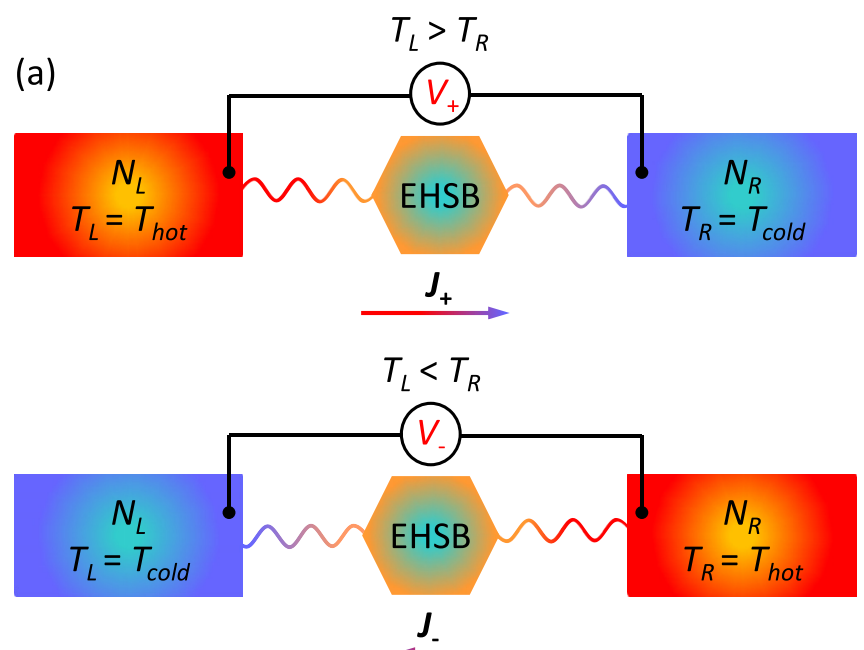

(b)

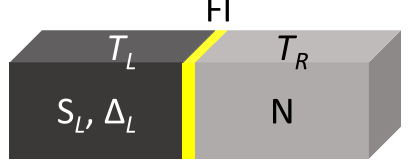

$\mathrm{FI}$

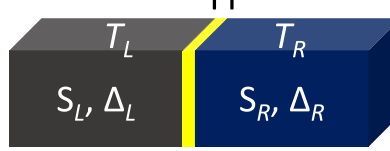

FIG. 1. (a) Sketch of two electronic reservoirs (either normal or superconducting) with different temperature-dependent densities of states $N_{L, R}$ residing at temperature $T_{L, R}$. The reservoirs are coupled to an element that breaks electron-hole symmetry. This leads to a thermoelectric response and enhanced thermal rectification. $J_{+}$and $J_{-}$represent the heat flow in the forward $\left(T_{L}>T_{R}\right)$ and reverse $\left(T_{L}<T_{R}\right)$ thermal-bias configuration, respectively, while $V_{+}$and $V_{-}$denote the corresponding thermovoltages developed across the system. (b) Prototypical ferromagnetic insulator (FI)-based superconductor $\left(S_{L}\right)$-normal metal $(N)$ and $(c)$ superconductor $\left(S_{L}\right)$-superconductor $\left(S_{R}\right)$ tunnel junctions. The $\mathrm{FI}$ is inserted in the structures as a tunnel barrier so as to both induce a spin splitting in the density of states of the superconductor and provide a spin filtering effect. $\Delta_{L}\left(\Delta_{R}\right)$ denotes the energy gap in $\mathrm{S}_{L}\left(\mathrm{~S}_{R}\right)$.

electrodes are electrically coupled via an EHSB mechanism. The latter gives rise to a thermoelectric response in the system and to a thermovoltage amplitude $V_{+}\left(V_{-}\right)$developed for $T_{L}>T_{R}\left(T_{L}<T_{R}\right)$. The heat current flowing in the forward thermal-bias configuration is denoted with $J_{+}$, whereas $J_{-}$denotes the one flowing in the reverse thermalbias configuration. As we shall show, the presence of EHSB in the system yields a self-amplification of thermal rectification efficiency up to unparalleled values for suitable parameters of the structure and proper thermal bias conditions. The EHSB mechanism can be achieved by placing an S-FI building block in two possible configurations: $S_{L}$ FIN [Fig. 1(b)] and $S_{L}$ FIS $_{R}$ [Fig. 1(c)] tunnel junctions. The presence of the FI layer yields both spin splitting of the density of states in $S_{L}$ and spin filtering at the barrier. The combination results in the EHSB mechanism. ${ }^{28-34}$

The interaction between the spin of conducting electrons in the superconductor and the localized magnetic moments in the adjacent FI leads to an effective exchange interaction $\left(h_{\text {exc }}\right)$ in the superconductor. This field decays away from the S/FI interface over the superconducting coherence length $\xi_{0}{ }^{35}$ Yet, we assume that the superconducting layer thickness is smaller than $\xi_{0}$, so that the induced $h_{\text {exc }}$ in the superconductor by FI is spatially homogeneous. In this situation, the spin-dependent normalized density of states of the superconductor is simply given by $N^{\uparrow, \downarrow}(E)=\frac{1}{2}\left|\operatorname{Re}\left[\frac{E+i \Gamma \pm h_{\mathrm{exc}}}{\sqrt{\left(E+i \Gamma \pm h_{\mathrm{exc}}\right)^{2}-\Delta^{2}}}\right]\right|$, where $\Gamma$ is the Dynes parameter and $\Delta$ is the superconducting gap, which depends on $T$ and $h_{\text {exc }}$ via the self-consistency equation $\ln \left(\frac{\Delta_{0}}{\Delta}\right)=\int_{0}^{h \omega_{D}} d E \frac{f_{+}(E)+f_{-}(E)}{\sqrt{E^{2}+\Delta^{2}}}$, where $f_{ \pm}(E)=\left\{1+\exp \left[\frac{1}{k_{B} T}\left(\sqrt{E^{2}+\Delta^{2}}\right.\right.\right.$ $\left.\left.\left.\mp h_{e x c}\right)\right]\right\}^{-1}, \omega_{D}$ is the Debye frequency of the superconductor, $\Delta_{0}$ $=1.764 k_{B} T_{c}$ is the zero-temperature, zero-exchange field superconducting pairing potential, $T_{c}$ is the critical temperature, and $k_{B}$ is the Boltzmann constant. The parameter $\Gamma$ accounts for the broadening of the coherent peaks in the density of states due to inelastic scattering, and for an ideal superconductor, $\Gamma \rightarrow 0^{+}{ }^{36}$ In all the calculations, we set $\Gamma=10^{-4} \Delta_{0}$, unless differently stated. We are interested in both the DC charge and electronic heat currents through the junctions, which are given by ${ }^{30} I=\frac{1}{e R_{T}} \int_{-\infty}^{\infty} d E\left[N_{+}+P N_{-}\right]\left[f_{L}\left(V, T_{L}\right)-f_{R}\left(T_{R}\right)\right]$ and $J=\frac{1}{e R_{T}} \int_{-\infty}^{\infty} d E(E+e V)\left[N_{+}+P N_{-}\right]\left[f_{L}\left(V, T_{L}\right)-f_{R}\left(T_{R}\right)\right]$, respectively. Here, $R_{t}$ is the normal-state tunneling resistance of the junction, $N_{ \pm}=\left(N_{L}^{\uparrow} N_{R}^{\uparrow} \pm N_{L}^{\downarrow} N_{R}^{\downarrow}\right.$, and $0 \leq P \leq 1$ is the barrier spin polarization provided by the FI layer. ${ }^{37}$ Moreover, $f_{L}\left(V, T_{L}\right)=[1+\exp [(E+\mathrm{eV}) /$ $\left.\left.k_{B} T_{L}\right]\right]^{-1}$ and $f_{R}\left(T_{R}\right)=\left[1+\exp \left(E / k_{B} T_{R}\right)\right]^{-1}$ are the equilibrium quasiparticle distribution functions, and $e$ is the electron charge. In principle, the expressions for both currents may contain additional phase-dependent contributions due to the Josephson coupling present in the junction. ${ }^{4,53}$ However, since our system exhibits a thermovoltage across the junction, the phase becomes time-dependent, thereby not contributing to DC transport. It is also worth emphasizing that the values of both spin polarization and spin splitting can be extracted from experiments, as demonstrated in several works. ${ }^{39-48}$

We assume that both electronic reservoirs are thermalized with well-defined temperatures $T_{L, R}$. In the forward thermal bias configuration [see Fig. 1(a)], a thermal gradient is intentionally created at the junction by setting $T_{L}=T_{\text {hot }}>T_{R}=T_{\text {cold }}$, which leads to a total heat flux $J_{+}$through the system. In the reverse thermal bias configuration, the heat gradient is inverted so that $T_{L}=T_{\text {cold }}<T_{R}=T_{\text {hot }}$, yielding a total heat current $J_{-}$flowing from the right to the left electrode. The electronic thermal rectification efficiency is defined as $R(\%)=100$ $\times\left(\left|J_{+}\right|-\left|J_{-}\right|\right) /\left|J_{-}\right|$, such that the absence of heat rectification corresponds to $R=0$ and $R>0$ indicates a preferential heat flow from the left to the right side of the junction. In order to determine the thermal rectification, for example, in the forward thermal bias configuration, for arbitrary $T_{L}>T_{R}$, we need first to determine the thermovoltage $V_{+}$across the junction by solving the equation $I\left(V_{+}, T_{L}, T_{R}\right)=0$. Then, the obtained thermovoltage is used to compute the corresponding heat current $J_{+}\left(V_{+}, T_{L}, T_{R}\right)$ flowing through the junction. In the reverse thermal bias condition, $T_{R}>T_{L}$, the same procedure is performed to determine $V_{-}$and $J_{-}$. Following this procedure, we determine $R$ in both junction setups depicted in Figs. 1(b) and 1(c).

It is instructive to start our discussion by analyzing the setup of Fig. 1(b), the $S_{L}$ FIN superconducting tunnel junction. A thermal bias across the structure leads to a thermovoltage $V_{ \pm}$, which depends on the sign of the thermal gradient itself, and stems from EHSB in the junction $^{49}$ [see Fig. 1(a)]. In particular, the thermovoltage $V_{+}$vs $T_{L}$ (at $T_{R}=0.01 T_{c}$ ) is shown in the left panel of Fig. 2(a), whereas $V_{-}$vs $T_{R}$ (at $T_{L}=0.01 T_{c}$ ) is shown in the right panel of the same figure, both evaluated at $P=1$ for selected values of the exchange field $h_{\text {exc }}$. Besides the substantial difference between the thermovoltage amplitudes $V_{+}$ and $V_{-}$for the same exchange field, $V_{+}$is a non-monotonic function of $T_{L}$, vanishing when the superconducting pairing potential goes to zero, while $V_{-}$monotonically increases with $T_{R}$, saturating at the 
(a)

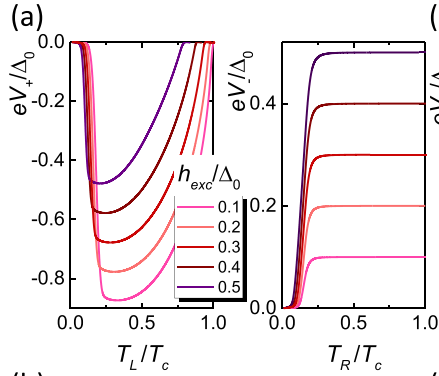

(b)

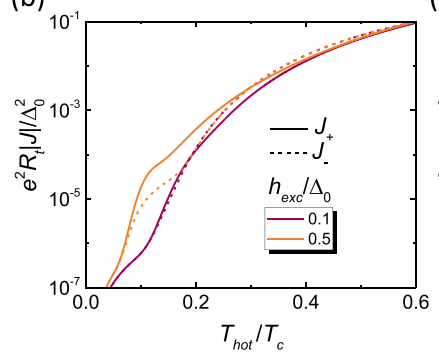

(c)

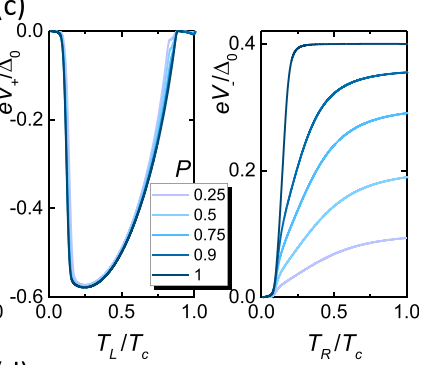

(d)

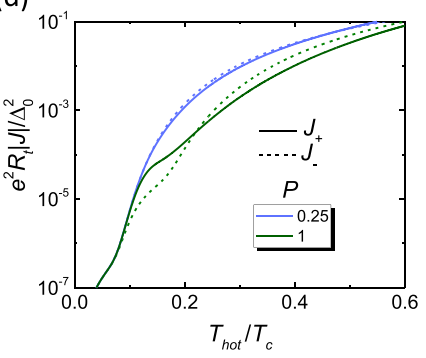

FIG. 2. (a) Thermovoltage $V_{+}$vs $T_{L}$ (left panel) and $V_{-}$vs $T_{R}$ (right panel) calculated for an $S_{L}$ FIN junction at $P=1$ and $T_{R}=0.01 T_{c}$ (left panel) and $T_{L}=0.01 T_{c}$ (right panel) for different values of the exchange field $h_{\text {exc. }}$ (b) Absolute value of the heat current $|J|$ vs $T_{\text {hot }}$ flowing through an $\mathrm{S}_{L}$ FIN junction calculated at $P=1$ and $T_{\text {cold }}=0.01 T_{c}$ for two selected values of $h_{\text {exc. }}$. (c) $V_{+}$vs $T_{L}$ (left panel) and $V_{-}$vs $T_{R}$ (right panel) calculated for an $\mathrm{S}_{L} \mathrm{FIN}$ junction at $h_{\text {exc }}=0.4 \Delta_{0}$ and $T_{R}=0.01 T_{c}$ (left panel) and $T_{L}=0.01 T_{c}$ (right panel) for different values of barrier polarization $P$. (d) Absolute value of the heat current $|J|$ vs $T_{\text {hot }}$ flowing through an $S_{L} F I N$ junction calculated at $h_{\text {exc }}=0.4 \Delta_{0}$ and $T_{\text {cold }}=0.01 T_{c}$ for two selected values of $P$.

asymptotic value $e V_{-}=h_{\text {exc }}$ at large temperature. ${ }^{49}$ This sizable difference between the thermovoltages has a direct impact on the corresponding heat currents $J_{ \pm}$flowing through the junction. These are shown in Fig. 2(b) for two given values of the exchange field. For the larger value of $h_{\text {exc }}$, the difference between the forward and reverse heat currents is increased, thereby leading to enhanced thermal rectification in the $\mathrm{S}_{L}$ FIN junction. This difference appears to be particularly pronounced for $0.1 T_{c} \lesssim T_{\text {hot }} \lesssim 0.2 T_{c}$, which is indeed the temperature range where the maximum thermovoltage amplitudes develop across the junction [cf. Fig. 2(a)].

The left panel of Fig. 2(c) displays $V_{+}$vs $T_{L}$ (at $T_{R}=0.01 T_{c}$ ), while the right one shows $V_{-}$vs $T_{R}$ (at $T_{L}=0.01 T_{c}$ ), both calculated at $h_{\text {exc }}=0.4 \Delta_{0}$ for different values of $P$. The thermovoltage $V_{+}$is negligibly affected by the polarization. By contrast, $V_{-}$is strongly affected by $P$ and becomes larger by increasing the barrier polarization. These different behaviors can be understood by noticing that $P$ changes only the effective barrier transmissivity and not the spectrum of the superconductor as $h_{\text {exc }}$ does [cf. Fig. 2(a)]. When $S_{L}$ is heated ( $V_{+}$situation), charge transport is weakly affected by interface transmissivity (i.e., by $P$ ) because of the large impedance provided by the gapped density of states. By contrast, $P$ mostly determines the charge current in the $V_{-}$ situation, because the $\mathrm{N}$ electrode has a large density of states at the Fermi energy, and, hence, very low impedance. Also, in the present case of finite barrier polarization, the large difference in thermovoltage amplitudes deeply affects the corresponding heat currents flowing through the structure, as shown in Fig. 2(d) for two selected values of $P$.

A large barrier polarization $(P \sim 1)$ strongly enhances the difference between forward and reverse heat currents, therefore leading to a sizable heat diode effect in the junction.

In Fig. 3, we show the rectification efficiency for the $S_{L F I N}$ junction. Specifically, Fig. 3(a) shows the heat rectification efficiency $R$ vs $T_{\text {hot }}$ calculated for zero barrier polarization at $T_{\text {cold }}=0.01 T_{c}$ for different values of $h_{\text {exc }}$. The absence of spin polarization at the barrier leads to a zero thermoelectric voltage. The increase in $h_{\text {exc }}$ yields a slight reduction of $R$ compared to that of a conventional SIN tunnel junction (i.e., for $h_{\text {exc }}=0$ ), ${ }^{6,18,19}$ allowing us to obtain a maximum rectification efficiency of $\sim 22 \%$ for $h_{\text {exc }}=0.5 \Delta_{0}$ at $T_{h o t} \sim 0.5 T_{c}$. The above heat rectification reduction stems from a larger contribution to the heat transport for electrons with energy close to the Fermi level at large $h_{\text {exc }}$. This contribution restores the thermal symmetry of the junction and, hence, reduces the heat rectification. In addition, there are additional features appearing in the rectification characteristics at higher temperatures, which occur when superconductivity is quenched due to the presence of a finite exchange field. In short, Fig. 3(a) demonstrates that spin splitting alone cannot improve thermal rectification with respect to conventional superconducting junctions.

However, if the barrier spin polarization is finite, the situation changes drastically. The role of finite $h_{\text {exc }}$ at $P=1$ is shown in Fig. 3(b). In particular, the thermoelectric response of the junction is increased in the presence of the high exchange field, allowing us to obtain both large heat rectification in the forward thermal-bias configuration for $h_{\text {exc }}=0.5 \Delta_{0}$ (i.e., up to $\sim 290 \%$ ) and a sizable contribution in the reverse thermal-bias configuration (around $\sim-55 \%$ for $h_{\text {exc }}=0.3 \Delta_{0}$ ). All the above results prove that the presence of EHSB mechanisms in the $S_{L}$ FIN junction leads to a substantial amount of self-amplification of the heat diode efficiency, which now yields values
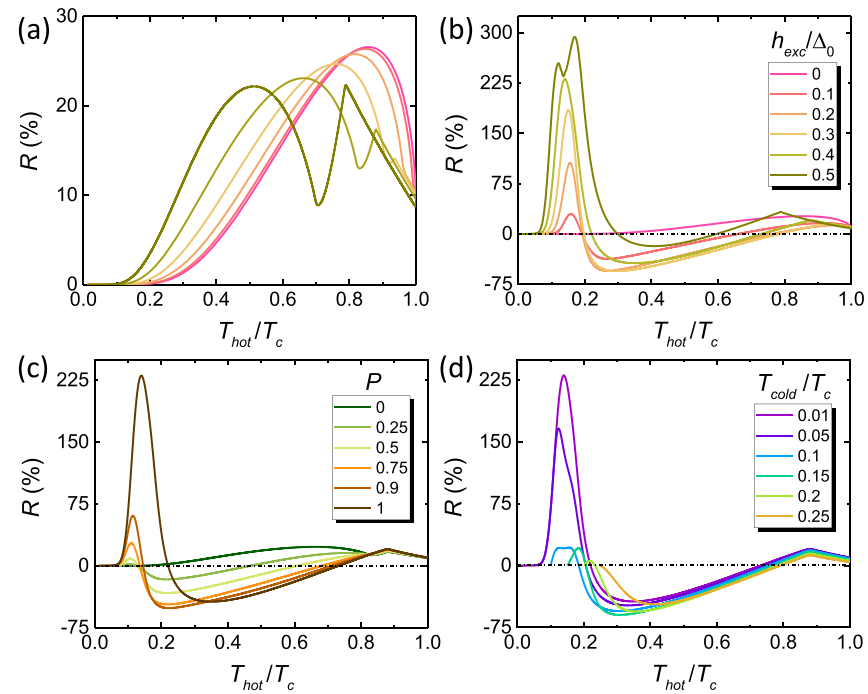

FIG. 3. Thermal rectification coefficient $R$ for the $S_{L}$ FIN junction. (a) Thermal rectification coefficient $R$ vs $T_{\text {hot }}$ calculated at $T_{\text {cold }}=0.01 T_{c}$ and $P=0$ for different values of the exchange field $h_{\text {exc }}$. (b) The same as in panel (a) but for barrier polarization $P=1$. (c) $R$ vs $T_{\text {hot }}$ calculated at $T_{\text {cold }}=0.01 T_{c}$ and $h_{\text {exc }}=0.4 \Delta_{0}$ for a few values of $P$. (d) $R$ vs $T_{\text {hot }}$ calculated at $h_{e x c}=0.4 \Delta_{0}$ and $P=1$ for several values of $T_{\text {cold. }}$. Dashed lines indicate $R=0$. 
that are larger by more than a factor of $\sim 10$ than those typically achievable in conventional superconducting tunnel junctions. ${ }^{6,18,19}$

The impact of a finite barrier polarization at $h_{\text {exc }}=0.4 \Delta_{0}$ is displayed in Fig. 3(c) for different values of $P$. The effect is similar to that caused by an increasing exchange field [see Fig. 3(c)] and shows the relevance of a large spin polarization in order to achieve a sizable heat rectification. For instance, $R$ turns out to be suppressed by more than a factor of $\sim 20$ if $P$ is reduced down to $50 \%$. Finally, the effect of the smaller temperature $T_{\text {cold }}$ on $R$ is displayed in Fig. 3(d) as a function of $T_{\text {hot }}$. We notice, in particular, the strong suppression of $R$ occurring by increasing $T_{\text {cold }}$ : for instance, $R$ is suppressed by roughly one order of magnitude at $T_{\text {cold }}=0.1 T_{c}$. This emphasizes the requirement of a sufficiently low $T_{\text {cold }}$ in order to achieve large rectification effects.

We now discuss the heat diode effect in the junction setup sketched in Fig. 1(c). For simplicity, we assume that the two superconductors are identical such that they have the same zero-temperature and zero-exchange field energy gap $\Delta_{0}$. To maximize the thermal asymmetry, we also assume that only the density of states of the left electrode $\left(N_{L}\right)$ is affected by $h_{\text {exc }}$ so as to break the thermal symmetry of the system. Such an asymmetry can be achieved by inserting a very thin non-magnetic oxide layer at the $F I / S_{R}$ interface. ${ }^{40,42}$ This implies that $N_{R}^{\uparrow}=N_{R}^{\downarrow}$. We now define the rectification efficiency as $R=100$ $\times\left(\left|J_{-}\right|-\left|J_{+}\right|\right) /\left|J_{+}\right|$so as to easily compare the two different junction setups since, as we shall show, $J_{-}$is typically much larger than $J_{+}$in the $S_{L} \mathrm{FIS}_{R}$ junction. Figures 4(a) and 4(b) show the impact of a finite $h_{\text {exc }}$ on $R$ at $P=1$. In particular, we note the very large thermal rectification, which can be obtained, up to $\sim 5 \times 10^{4} \%$ for $h_{e x c}=0.5 \Delta_{0}$ at $T_{\text {hot }} \simeq 0.15 T_{c}$. This value is $\sim 250$ times larger than the one achievable in conventional all-superconducting tunnel junctions made with superconductors with different energy gaps. ${ }^{6,18}$ Yet, even for moderate $h_{\text {exc }}$ values (i.e., $h_{\text {exc }}=0.2 \Delta_{0}$ ), $R$ can reach values as large as $10^{4} \%$ in the suitable $T_{\text {hot }}$ range. The above results demonstrate the effectiveness of $S_{L}$ FIS $_{R}$ tunnel junctions to achieve very high thermal rectification efficiency.

The role of $P$ is displayed in Figs. 4(c) and 4(d), which reveal the increased robustness of the $S_{L}$ FIS $_{R}$ setup with respect to the $S_{L}$ FIN one in terms of limited barrier polarization. For example, for a moderate spin polarization, $P=75 \%$, we still get a sizable $R \simeq 1370 \%$, which is about two times larger than the maximum value achievable in conventional SIS tunnel junctions. ${ }^{6,18}$ Nowadays, for instance, state-of-the-art ferromagnetic europium $(\mathrm{Eu})$ chalcogenide tunnel barriers can provide spin polarization close to $100 \%,{ }^{50}$ which would make thermal rectification efficiencies larger than $\sim 7.5 \times 10^{3} \%$ readily available in superconducting tunnel junction setups operating at cryogenic temperatures.

The impact of non-idealities of the junction is shown in Fig. 4(e), where $R$ is plotted against $T_{\text {hot }}$ for a few selected values of $\Gamma$. From a quantitative point of view, thermal rectification efficiency turns out to be less effective for the larger value of $\Gamma$. In particular, for a sizable $\Gamma=10^{-2} \Delta_{0}$, the maximum of $R$ is reduced down to $\sim 1140 \%$ at $T_{\text {hot }} \simeq 0.25 T_{c}$. This fact emphasizes the requirement of high-quality tunnel junctions in order to preserve a substantial heat diode effect. Finally, Fig. 4(f) shows how $T_{\text {cold }}$ affects the rectification efficiency. Analogous to the $\mathrm{S}_{L}$ FIN setup, increasing $T_{\text {cold }}$ deeply suppresses the $R$ coefficient, although in a reduced way. In particular, for $T_{\text {cold }}=0.1 T_{c}$, the rectification efficiency turns out to be decreased by almost a factor (a)

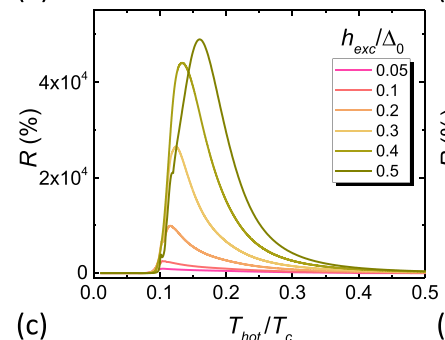

(b)

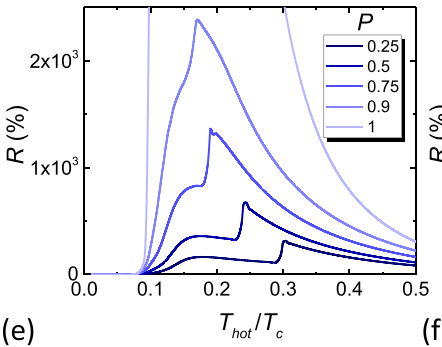

(d) $\quad T_{\text {hot }} / T_{c}$
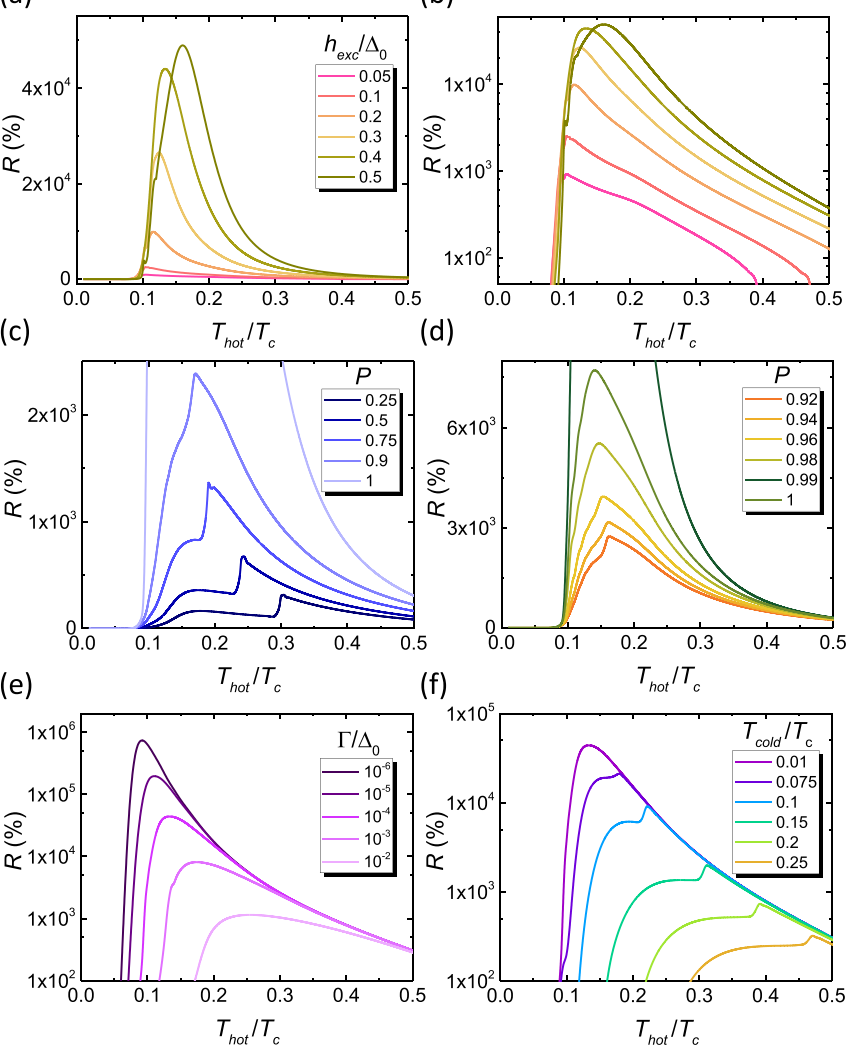

FIG. 4. Thermal rectification coefficient $R$ for the $S_{L} F I S_{R}$ junction. (a) $R$ vs $T_{\text {hot }}$ calculated at $T_{\text {cold }}=0.01 T_{c}$ and $P=1$ for different values of the exchange field $h_{\text {exc }}$. (b) The same as in panel (a) but shown on a logarithmic scale. (c) $R$ vs $T_{\text {hot }}$ calculated at $T_{\text {cold }}=0.01 T_{c}$ and $h_{\text {exc }}=0.4 \Delta_{0}$ for a few values of $P$. (d) $R$ vs $T_{\text {hot }}$ calculated at $T_{\text {cold }}=0.01 T_{c}$ and $h_{\text {exc }}=0.4 \Delta_{0}$ for several high values of barrier polarization in the range of $0.92 \leq P \leq 1$. (e) $R$ vs $T_{\text {hot }}$ calculated at $T_{\text {cold }}=0.01 T_{c}, h_{\text {exc }}=0.4 \Delta_{0}$, and $P=1$ for a few values of $\Gamma$. (f) $R$ vs $T_{\text {hot }}$ calculated at $h_{e x c}=0.4 \Delta_{0}$ and $P=1$ for different values of $T_{\text {cold. }}$.

of 5 with respect to the lowest temperature, suggesting that the $\mathrm{S}_{L} \mathrm{FIS}_{R}$ junction is more efficient as a heat diode at higher temperatures than the $S_{L}$ FIN setup.

In summary, we have demonstrated theoretically the occurrence of an unparalleled thermal diode effect in superconducting tunnel junctions with ferromagnetic insulators. In particular, thermal rectification efficiencies above $\sim 5 \times 10^{4} \%$ could be achieved for realistic material parameters in a suitable temperature range. Such a heat rectifier efficiency exceeds by a factor of $\sim 250$, the one obtained with conventional superconducting tunnel junctions. Ideal materials for the heat rectifier are europium chalcogenide layers ( $\mathrm{EuO}$ and $\mathrm{EuS}$ ), for which values of $P$ ranging from $80 \%$ up to $\sim 100 \%$ have been reported, ${ }^{37,40,42,51-55}$ in combination with $\mathrm{Al}$ superconducting thin films. ${ }^{46-48}$ Yet, very large spin-filtering has been reported in GdN barriers as well, ${ }^{56-58}$ with polarizations as large as $97 \%$ at low temperature. Our finding might be relevant for enhanced control of heat current in phase-coherent caloritronic devices ${ }^{4,5}$ as well as for general thermal management of nanoscale quantum circuits. 
The authors acknowledge the European Union's Horizon 2020 research and innovation program under Grant Agreement No. 800923-SUPERTED and the Spanish Ministerio de Ciencia e Innovacion (MICINN) through Project No. FIS2017-82804-P, for partial financial support.

\section{DATA AVAILABILITY}

The data that support the findings of this study are available from the corresponding author upon reasonable request.

\section{REFERENCES}

${ }^{\mathbf{1}}$ N. A. Roberts and D. Walker, Int. J. Therm. Sci. 50, 648 (2011).

${ }^{2}$ B. Li, L. Wang, and G. Casati, Phys. Rev. Lett. 93, 184301 (2004).

${ }^{3}$ F. Giazotto, T. T. Heikkilä, A. Luukanen, A. M. Savin, and J. P. Pekola, Rev. Mod. Phys. 78, 217 (2006).

${ }^{4}$ A. Fornieri and F. Giazotto, Nat. Nanotechnol. 12, 944 (2017).

${ }^{5}$ M. Martínez-Pérez, P. Solinas, and F. Giazotto, J. Low Temp. Phys. 175, 813 (2014).

${ }^{6}$ A. Fornieri, M. J. Martínez-Pérez, and F. Giazotto, AIP Adv. 5, 053301 (2015).

${ }^{7}$ L.-A. Wu and D. Segal, Phys. Rev. Lett. 102, 095503 (2009).

${ }^{8}$ D. Segal, Phys. Rev. Lett. 100, 105901 (2008).

${ }^{9}$ B. Li, L. Wang, and G. Casati, Appl. Phys. Lett. 88, 143501 (2006).

${ }^{10}$ M. Terraneo, M. Peyrard, and G. Casati, Phys. Rev. Lett. 88, 094302 (2002).

${ }^{11}$ R. López and D. Sánchez, Phys. Rev. B 88, 045129 (2013).

${ }^{12}$ J. Ren and J.-X. Zhu, Phys. Rev. B 87, 165121 (2013).

${ }^{13}$ L. Bours, B. Sothmann, M. Carrega, E. Strambini, A. Braggio, E. M. Hankiewicz, L. W. Molenkamp, and F. Giazotto, Phys. Rev. Appl. 11, 044073 (2019).

${ }^{14} \mathrm{~T}$. Ruokola and T. Ojanen, Phys. Rev. B 83, 241404 (2011).

${ }^{15}$ T. Ruokola, T. Ojanen, and A.-P. Jauho, Phys. Rev. B 79, 144306 (2009).

${ }^{16}$ D. M.-T. Kuo and Y-C Chang, Phys. Rev. B 81, 205321 (2010).

${ }^{17}$ A. Fornieri, M. J. Martínez-Pérez, and F. Giazotto, Appl. Phys. Lett. 104, 183108 (2014).

${ }^{18}$ M. Martínez-Pérez and F. Giazotto, Appl. Phys. Lett. 102, 182602 (2013).

${ }^{19}$ F. Giazotto and F. Bergeret, Appl. Phys. Lett. 103, 242602 (2013).

${ }^{20} \mathrm{D}$. Goury and R. Sánchez, Appl. Phys. Lett. 115, 092601 (2019).

${ }^{21}$ P. Ben-Abdallah and S.-A. Biehs, Appl. Phys. Lett. 103, 191907 (2013).

${ }^{22}$ M. J. Martínez-Pérez, A. Fornieri, and F. Giazotto, Nat. Nanotechnol. 10, 303 (2015).

${ }^{23}$ J. Senior, A. Gubaydullin, B. Karimi, J. T. Peltonen, J. Ankerhold, and J. P. Pekola, Commun. Phys. 3, 40 (2020).

${ }^{24}$ R. Scheibner, M. König, D. Reuter, A. D. Wieck, C. Gould, H. Buhmann, and L. W. Molenkamp, New J. Phys. 10, 083016 (2008).

${ }^{25}$ C. W. Chang, D. Okawa, A. Majumdar, and A. Zettl, Science 314, 1121 (2006).

${ }^{26}$ W. Kobayashi, Y. Teraoka, and I. Terasaki, Appl. Phys. Lett. 95, 171905 (2009).
${ }^{27}$ H. Tian, D. Xie, Y. Yang, T.-L. Ren, G. Zhang, Y.-F. Wang, C.-J. Zhou, P.-G. Peng, L.-G. Wang, and L.-T. Liu, Sci. Rep. 2, 523 (2012).

${ }^{28}$ F. S. Bergeret, M. Silaev, P. Virtanen, and T. T. Heikkilä, Rev. Mod. Phys. 90, 041001 (2018).

${ }^{29}$ T. T. Heikkilä, M. Silaev, P. Virtanen, and F. S. Bergeret, Prog. Surf. Sci. 94, 100540 (2019).

${ }^{30}$ A. Ozaeta, P. Virtanen, F. Bergeret, and T. Heikkilä, Phys. Rev. Lett. 112, 057001 (2014).

${ }^{31}$ P. Machon, M. Eschrig, and W. Belzig, Phys. Rev. Lett. 110, 047002 (2013).

${ }^{32}$ P. Machon, M. Eschrig, and W. Belzig, New J. Phys. 16, 073002 (2014).

${ }^{33}$ S. Kolenda, M. J. Wolf, and D. Beckmann, Phys. Rev. Lett. 116, 097001 (2016).

${ }^{34}$ J. Linder and J. W. Robinson, Nat. Phys. 11, 307 (2015).

${ }^{35}$ T. Tokuyasu, J. A. Sauls, and D. Rainer, Phys. Rev. B 38, 8823 (1988).

${ }^{36}$ R. Dynes, J. Garno, G. Hertel, and T. Orlando, Phys. Rev. Lett. 53, 2437 (1984).

37J. S. Moodera, T. S. Santos, and T. Nagahama, J. Phys.: Condens. Matter 19, 165202 (2007).

${ }^{38}$ F. Giazotto and M. J. Martínez-Pérez, Nature 492, 401 (2012).

${ }^{39}$ R. Meservey and P. Tedrow, Phys. Rep. 238, 173 (1994).

${ }^{40}$ X. Hao, J. Moodera, and R. Meservey, Phys. Rev. B 42, 8235 (1990).

${ }^{41}$ B. Li, G.-X. Miao, and J. S. Moodera, Phys. Rev. B 88, 161105 (2013).

${ }^{42}$ B. Li, N. Roschewsky, B. A. Assaf, M. Eich, M. Epstein-Martin, D. Heiman, M. Münzenberg, and J. S. Moodera, Phys. Rev. Lett. 110, 097001 (2013).

${ }^{43}$ Y. Xiong, S. Stadler, P. Adams, and G. Catelani, Phys. Rev. Lett. 106, 247001 (2011).

${ }^{44}$ T. Liu, J. Prestigiacomo, and P. Adams, Phys. Rev. Lett. 111, 027207 (2013).

${ }^{45}$ M. Wolf, C. Sürgers, G. Fischer, and D. Beckmann, Phys. Rev. B 90, 144509 (2014).

${ }^{46}$ E. Strambini, V. Golovach, G. De Simoni, J. Moodera, F. Bergeret, and F. Giazotto, Phys. Rev. Mater. 1, 054402 (2017).

${ }^{47}$ G. D. Simoni, E. Strambini, J. S. Moodera, F. S. Bergeret, and F. Giazotto, Nano Lett. 18, 6369 (2018).

${ }^{48}$ M. Rouco, S. Chakraborty, F. Aikebaier, V. N. Golovach, E. Strambini, J. S. Moodera, F. Giazotto, T. T. Heikkilä, and F. S. Bergeret, Phys. Rev. B 100, 184501 (2019).

${ }^{49}$ F. Giazotto, P. Solinas, A. Braggio, and F. S. Bergeret, Phys. Rev. Appl. 4, 044016 (2015).

${ }^{50}$ J. S. Moodera, G.-X. Miao, and T. S. Santos, Phys. Today 63(4), 46 (2010).

${ }^{51}$ J. Moodera, X. Hao, G. Gibson, and R. Meservey, Phys. Rev. Lett. 61, 637 (1988).

${ }^{52}$ T. S. Santos and J. S. Moodera, Phys. Rev. B 69, 241203 (2004).

${ }^{53}$ J. Moodera, R. Meservey, and X. Hao, Phys. Rev. Lett. 70, 853 (1993).

${ }^{54}$ T. Santos, J. Moodera, K. Raman, E. Negusse, J. Holroyd, J. Dvorak, M. Liberati, Y. Idzerda, and E. Arenholz, Phys. Rev. Lett. 101, 147201 (2008).

${ }^{55}$ G.-X. Miao and J. S. Moodera, Appl. Phys. Lett. 94, 182504 (2009).

${ }^{56}$ K. Senapati, M. G. Blamire, and Z. H. Barber, Nat. Mater. 10, 849 (2011).

${ }^{57}$ A. Pal, K. Senapati, Z. Barber, and M. Blamire, Adv. Mater. 25, 5581 (2013).

${ }^{58}$ A. Pal, Z. Barber, J. Robinson, and M. Blamire, Nat. Commun. 5, 3340 (2014). 